\title{
Sobre el origen y difusión de la siderurgia protohistórica en el Levante español: el conjunto minero-metalúrgico del término municipal de Seno
} (Bajo Aragón)

\author{
A. Martín-Costea*, V. López-Serrano* y A. Gabaldón-García** \\ Resumen Se estudiaron dos minas a cielo abierto y otra subterránea con restos óseos, cerámica \\ ibérica, etc. aterrada para su sellado. Igualmente, se analizaron los restos de un horno de \\ reducción. Los análisis de escorias férreas de tres yacimientos cercanos ayudaron a \\ comprender su formación en el horno, los minerales que intervinieron en el proceso, las \\ temperaturas de trabajo, etc. Se estudiaron dos objetos férreos, uno del Hierro I y otro \\ ibérico y se determinó el sistema operativo de los hornos de reducción ibéricos. Todo ello \\ permitió conocer cómo se desarrollaban los procesos minero-metalúrgicos férreos en el \\ Levante español entre los siglos V al III a.C. correspondiendo con la difusión de la \\ siderurgia en el área.
}

Palabras clave Siderurgia. Instalaciones mineras. Horno de reducción. Cultura ibérica levantina. Villa de Seno (Bajo Aragón-España).

\section{Origin and diffusion of the protohistoric siderurgy in the East of Spain: the miner-metallurgical combined of the council term of Seno (Bajo Aragón, Spain)}

\begin{abstract}
Two mines have been studied opencast, also a low level mine with bony remains, Iberian ceramics, etc., which was pulled down for its sealing off. Likewise, the remains of a reduction furnace were also analysed. The analysis of the ferrous slag obtained in three nearby deposits, has helped to understand its formation in the furnace, as well as the ores that were used during the process, the work temperatures, etc. The characterization of two ferrous objects was carried out, one was an Iberian period object and the other one from the First Age of Iron. The operating system of the Iberian reduction furnaces has been determined too. The conclusions permited to know the development of a minermetallurgical process, between the V and III century B.C. corresponding with the diffusion of siderurgy in the studied area.
\end{abstract}

Keywords Siderurgy. Mining facilities. Reduction furnace. Iberian levantine culture. Village of Seno (Bajo Aragón, Spain).

\section{INTRODUCCIÓN}

Ya en la antigüedad, algunos autores clásicos elogiaron la excelencia de la producción férrea obtenida por los celtíberos. En el actual Bajo Aragón los objetos férreos más antiguos remiten al siglo VII a.C., siendo también escasos en la centuria siguiente. La difusión de la metalurgia del hierro tuvo su principio coincidiendo con el de la Cultura Ibérica, a partir del siglo $\mathrm{V}$ a.C. A estos momentos de difusión de la primitiva siderurgia bajoaragonesa corresponde el trabajo que a continuación se presenta.

\section{INSTALACIONES Y OTROS RESTOS MINERO- METALÚRGICOS}

En la cuenca media del río Guadalope, en el término municipal de Seno, se localiza el conjunto minero-metalúrgico que a continuación se describe.

(*) Centro Nacional de Investigaciones Metalúrgicas-CENIM-(CSIC). Avda. del Gregorio del Amo, $8.28040-M A D R I D$. Email: amcostea@cenim.csic.es / vlopez@cenim.csic.es

${ }^{* *}$ Instituto del Patrimonio Histórico Español -IPHE- (Ministerio de Educación, Cultura y Deporte). El Greco, 4. 28040-MADRID. araceli.gabaldon@iphe.mcu.es 
Mina de Valdestrada: Consiste en un corte en sentido E-O de la envolvente rocosa con lo que han quedado al descubierto varios filones longitudinales paralelos de mineral férrico con una altura máxima superior a $4 \mathrm{~m}$. El frente de explotación ocupa una anchura de aprox. $30 \mathrm{~m}$ y una altura máxima de unos $9 \mathrm{~m}$. El yacimiento mineral, al igual que los otros que más adelante se detallan (MDT-I y MDT-II) es una mineralización de goetita y en escasa proporción limonita, originada por rellenos ferruginosos kársticos entre carbonatos cretácicos (Cenomanense) ${ }^{[1]}$. La composición tipo del mineral de esta mina se ofrece en la tabla I.

Visto el mineral al microscopio óptico, se determinó que estaba constituido por goetita con estructura colomorfa, abundante ganga caliza y masas microcristalinas de limonita separadas por vetas de ganga.

Preparación minera MDT-I: En una loma cercana se localizó la preparación para una explotación similar a Valdestrada, consistente en un corte horizontal de la masa calcárea explanando y nivelando la superficie y dejando ver la alineación oscura de un filón de mineral férrico de 10,10 m de longitud y 2,20 $\mathrm{m}$ de anchura máxima. Analizada una muestra de este mineral, arrojó los resultados que se observan en la tabla I.

Mina subterránea MDT-II: A escasos metros hacia el sur de la preparación MDT-I se localizó la mina subterránea MDT-II. El acceso actual se realiza por dos aberturas en la roca, estando el interior casi colmatado de piedras y tierra. Se comprobó que la oquedad interior no era una zona de la que partían diversas galerías sino que se trataba de la parte superior de una gran cavidad abovedada subterránea excavada a pico. El diámetro aquí supera los $20 \mathrm{~m}$. El aterramiento de la explotación no fue

Tabla I. Análisis químico de minerales, \% en masa

Table I. Chemical analysis of minerals, \% in mass

\begin{tabular}{lcc}
\hline & Valdestrada & MDT-I \\
\hline $\mathrm{Fe}^{\text {total }}$ & 72,71 & 45,8 \\
$\mathrm{Mn}$ & $\leq 0,1$ & 0,026 \\
$\mathrm{MgO}$ & 0,033 & 0,12 \\
$\mathrm{CO}_{2}$ & 6,67 & 2,70 \\
$\mathrm{CaO}$ & 8,75 & 11,55 \\
$\mathrm{SiO}_{2}$ & 1,52 & 1,56 \\
$\mathrm{P}$ & 0,027 & 0,20 \\
$\mathrm{~S}$ & 0,022 & $<0,005$ \\
$\mathrm{Al}_{2} \mathrm{O}_{3}$ & $\leq 0,5$ & 0,45 \\
\hline
\end{tabular}

fortuito, sino intencionado. La zona a la que se puede acceder ahora es, por lo tanto, el hueco que ha quedado por el apelmazamiento del material de relleno durante más de dos milenios.

En el interior, se hallaron en superficie abundantes restos óseos de Bos taurus, Ovis aries-Capra hircus, Sus scrofa, Lepus capensis, etc., un fragmento de tronco de sabina o enebro quemado en un extremo, indicativo de que había servido como antorcha, y un fragmento de fondo anular y pared de un cuenco ibérico elaborado a torno con decoración de tres bandas pintadas al almagre al interior.

Horno de reducción MDT-III: En las inmediaciones de MDT-II un arroyamiento ha puesto al descubierto un horno excavado en el terreno con unas medidas, en la zona conservada, de 2,10 m de diámetro y una altura actual de $1,40 \mathrm{~m}$. Al interior se halla recubierto por una capa refractaria de arcilla de tono rojizo, endurecida por el fuego, con un espesor actual de $14 \mathrm{~cm}$. En los alrededores del horno se encuentran fragmentos de mineral férreo, algunas escorias y bloques de piedra calcárea; no se localizan fragmentos cerámicos. Los resultados del análisis del material refractario que recubre el interior del horno se ofrecen en la tabla II. En las inmediaciones del horno se halló una escoria cuyo análisis se recoge también en esta tabla.

También se localizaron escoriales siderúrgicos en varios poblados ibéricos de la zona denominados Vallipón, Monte Catma, Pico de La Ginebrosa, Santa Flora, y en dos puntos en la partida de Los Cabanes.

Vallipón es un poblado amurallado cuyos niveles inferiores remiten a los Campos de Urnas del Hierro tardíos, al que se superpone el yacimiento

Tabla II. Análisis químico del refractario y de la escoria del horno MDT-III, \% en masa

Table II. Chemical analysis of refractory slag of the oven MDT-III, \% in mass

\begin{tabular}{lcc}
\hline & Refractario & Escoria \\
\hline $\mathrm{Fe}^{\text {total }}$ & 2,66 & 7,0 \\
$\mathrm{Mn}$ & - & 0,020 \\
$\mathrm{MgO}$ & 0,56 & 0,64 \\
$\mathrm{CaO}$ & 43,97 & 12,79 \\
$\mathrm{C}$ & - & 0,085 \\
$\mathrm{SiO}_{2}$ & 15,1 & 55,40 \\
$\mathrm{P}$ & - & 0,063 \\
$\mathrm{~S}$ & - & $<0,005$ \\
$\mathrm{Al}_{2} \mathrm{O}_{3}$ & 4,78 & 21,65 \\
\hline
\end{tabular}


Sobre el origen y difusión de la siderurgia protohistórica en el Levante español: el conjunto minero-metalúrgico del término municipal de Seno (Bajo Aragón)

A. Martín-Costea, V. López-Serrano y A. Gabaldón-García

ibérico propiamente dicho, cuyos materiales cerámicos marcan un final de habitamiento datable entre finales del siglo III y principios del II a.C., quedando desde entonces deshabitado.

Las escorias recogidas se pueden agrupar en dos tipos claramente diferenciados: unas escasamente movilizadas, con abundantes poros -en algunos casos rellenos de arena, carbón vegetal o tierra- y tonalidades grises y anaranjadas en diversas zonas, apreciándose en algunos casos la granulometría de algunos componentes, y otras más movilizadas, de aspecto compacto y escasa y pequeña porosidad, color gris oscuro y densidad netamente más elevada que las descritas anteriormente, con aspecto muy fundido ${ }^{[2]}$. Un análisis representativo de las escorias poco movilizadas (o de baja temperatura) se muestra en la tabla III. El otro tipo de escorias, con aspecto muy fundido (de elevada temperatura) arrojó los resultados que muestra la misma tabla.

Al microscopio óptico, las escorias poco movilizadas mostraban granos muy abundantes de calcita que coexistían con masas de dolomita y limonita. En algunas zonas, se veían transformaciones parciales de limonita a hematita. También se apreciaban granos de cuarzo y calcita adicionales, con un tamaño relativamente homogéneo, lo que indica una molienda. El tamaño de dichos granos era de $250 \mu \mathrm{m}$, aproximadamente. También parecía que el mineral férrico estuviera molido con un tamaño de grano inferior a $1 \mathrm{~cm}$.

Las escorias de elevadas temperaturas estaban constituidas por granos redondeados y estructuras arborescentes de wustita entre la que se localizaban pequeñas masas de hierro metálico. En los bordes se veían algunas formas dendríticas de wustita.

Sobre algunas muestras de mineral y de las escorias poco movilizadas se realizaron análisis espectroscópicos, observándose la similitud de las gráficas en los picos de bario $(\mathrm{Ba})$, titanio $(\mathrm{Ti})$ y vanadio $(\mathrm{V})^{[1]}$.

Monte Catma es un gran poblado de cronología similar a Vallipón. Fuera del recinto de murallas se localizan considerables escoriales, sin duda, contemporáneos del yacimiento. Las escorias estudiadas de este yacimiento son, en todo, similares a las de Vallipón. Los valores analíticos de las mismas se muestran en la tabla III.

En la partida de Los Cabanes se localizan algunas concentraciones de escorias de escasa entidad, que parecen corresponder al período ibérico. De aquí, se analizó una escoria medianamente movilizada que arrojó el resultado que se observa en la tabla III.

Son muy escasas las muestras férreas conocidas de esta época en la zona estudiada, debido a que no se han llevado a cabo excavaciones arqueológicas en los yacimientos ibéricos. Una de ellas es un instrumento indeterminado aparecido en el poblado Las Terraceras I (Mas de las Matas), correspondiente a los Campos de Urnas del Hierro pero acusando aún influencias del Bronce Final. El aspecto externo del objeto manifiesta haber sufrido una severa degradación en el medio terroso. Los núcleos centrales de las probetas tomadas en dicho objeto eran unas masas isótropas uniformes de color gris verdoso claro que contenían cristales de cuarzo sin asimilar. Se apreció una estructura formada por

Tabla III. Análisis químico de las escorias de varios yacimientos arqueológicos de la zona, \% en masa

Table III. Chemical analysis of slags of several archaeologycal deposits, \% in mass

\begin{tabular}{lccccc}
\hline & \multicolumn{2}{c}{ Vallipón } & \multicolumn{2}{c}{ Monte Catma } & Los Cabanes I \\
\hline & Poco movilizada & Muy movilizada & Poco movilizada & Muy movilizada & Medianamente movilizada \\
\cline { 2 - 6 } $\mathrm{Fe}^{\text {total }}$ & 29,46 & 60,51 & 32 & 59,0 & 47,7 \\
$\mathrm{Mn}$ & 1,46 & 0,3 & - & - & 6,65 \\
$\mathrm{MgO}$ & 0,17 & 0,33 & 1,0 & 0,60 & 0,49 \\
$\mathrm{CaO}$ & 17,5 & 4,9 & 16,0 & 4,0 & 6,98 \\
$\mathrm{C}$ & 0,5 & 0,027 & 0,17 & 0,054 & 0,10 \\
$\mathrm{SiO}_{2}$ & 40,4 & 8,2 & 2,7 & 1,9 & 13,14 \\
$\mathrm{P}$ & 0,27 & 0,27 & - & - & 0,080 \\
$\mathrm{~S}$ & 0,029 & 0,068 & 0,033 & 0,063 & 0,044 \\
$\mathrm{Al}_{2} \mathrm{O}_{3}$ & $\leq 0,5$ & $\leq 0,5$ & 5,0 & 4,2 & 5,26 \\
\hline
\end{tabular}


yuxtaposición de agujas cristalinas alrededor de uno de los poros internos y la existencia de una estructura colomorfa muy acentuada que pudo haber sido provocada al obtener el objeto por un procedimiento de forja ${ }^{[3]}$. Un análisis por difracción de rayos $\mathrm{X}$, realizado sobre una muestra de esta pieza, manifestó que estaba constituida por, aproximadamente, un 46,6 \% en forma de $\mathrm{Fe}_{2} \mathrm{O}_{3}$ y un $29,4 \%$ de $\mathrm{Fe}_{3} \mathrm{O}_{4}$.

La otra muestra férrea procede del poblado ibérico Monte Catma. Se trata de un goterón de fundición metálico, informe, hallado entre las escorias extramuros del poblado. Las formas redondeadas y globulares parecen indicar que en su fusión se alcanzaron temperaturas muy elevadas. Preparada una probeta metalográfica de dicha muestra se observó grafito laminar en una matriz metálica de perlita con restos de cementita en zonas localizadas del producto.

\section{DISCUSIÓN}

El mineral de Valdestrada y de MDT es similar al de algunas explotaciones celtibéricas del Moncayo, con parecidos contenidos de hierro y de los otros componentes ${ }^{[4]}$.

La asociación del mineral de Valdestrada con el de las escorias escasamente movilizadas del poblado ibérico de Vallipón se ha determinado por similitud de los picos de $\mathrm{Ba}$, Ti y V, en análisis espectroscópicos realizado a tal fin.

La cronología de la mina MDT-II queda atestiguada por el fragmento de cuenco ibérico hallado en su interior procedente, sin duda, de los niveles más altos. Los restos óseos de diversas especies hallados en el interior de MDT-II parecen corresponder al momento de sellado de la cavidad. Al final de la explotación, en el período ibérico, la cavidad fue totalmente rellenada de tierra y piedras de los alrededores. La parte accesible de la mina es hoy el hueco que ha quedado por apelmazamiento.

Los hornos de reducción de hierro más antiguos de Europa, correspondientes a la Primera Edad del Hierro, eran una cubeta excavada en el suelo recubierta de material refractario, a la que se accedía durante la operación por su abertura superior. Durante la Segunda Edad del Hierro los hornos evolucionaron y adquirieron mayor altura ${ }^{[5]}$. La carga se hacía por su parte superior, aunque se dotaron de una abertura en la parte inferior por la que se podía sangrar la escoria de alta temperatura. Se alcanzaban temperaturas más elevadas (en la cuba entre 1.100 y $1.300^{\circ} \mathrm{C}$ ), suficientes para reducir el mineral pero sin llegar a la fusión. A esta tipología corresponde el horno MDT-III ${ }^{[5]}$.

Según se desprende de las escorias poco movilizadas de Vallipón y Monte Catma, que presentan ocluidas partículas de carbón vegetal, fue éste el combustible que se empleó en el proceso siderúrgico. Este carbón se obtenía de las masas boscosas inmediatas de encina, enebro y sabina.

Con respecto al horno MDT-III, se debe hacer notar que el escaso diámetro, el recubrimiento cerámico interno y la inexistencia de cerámica y arcillas en los alrededores indican que no se trata de un horno de cocer cerámica. Por el contrario, la existencia en sus inmediaciones de mineral de hierro, algunas escorias y piedra caliza, aparte de la morfología de la instalación, llevan a concluir que se trata de un horno de reducción de mineral férrico, probablemente de los más antiguos de la zona, cuando aún existía masa forestal en las inmediaciones de la mina de la que poder obtener las necesarias cantidades de carbón vegetal

Si se considera el análisis químico del recubrimiento cerámico interno del horno, el bajo contenido de sílice, normal de alúmina y elevado de caliza parecen indicar que no se trata de un material sílico-refractario idóneo. Para determinarlo se realizaron sobre la muestra cinco ensayos de reblandecimiento y fusión en atmósfera reductora (CO), que proporcionaron unos valores medios de temperatura de reblandecimiento de $1.595^{\circ} \mathrm{C}$, y de $1.629{ }^{\circ} \mathrm{C}$ para temperatura de fluidez. De ello se deduce que dicho recubrimiento pudo responder adecuadamente a las temperaturas que se debieron alcanzar en este horno.

En los escoriales, a simple vista se observan, fundamentalmente, dos tipos de escorias: unas, escasamente movilizadas de coloración gris y anaranjada, de baja densidad, procedentes de zonas del horno de baja temperatura y, otras, de coloración muy oscura y elevada densidad que tienen su origen en la zona del horno de temperaturas elevadas.

Al microscopio, las escorias de baja temperatura muestran que el mineral fue molido a un tamaño de grano de aproximadamente $1 \mathrm{~cm}$, y que al horno se añadieron -como fundente y fluidificante de la escoria- caliza, también molida a un tamaño menor, de aproximadamente $250 \mu \mathrm{m}$, y sílice.

La escoria del horno MDT-III, a la vista de los constituyentes en su correspondiente diagrama ternario, procede de una zona de elevada temperatura.

Las escorias de elevada temperatura presentan el aspecto externo de muy fundidas y son de color 
Sobre el origen y difusión de la siderurgia protohistórica en el Levante español: el conjunto minero-metalúrgico del término municipal de Seno (Bajo Aragón)

A. Martín-Costea, V. López-Serrano y A. Gabaldón-García

muy oscuro con pequeños y escasos poros, lo que se deduce también de su composición: wustita globular y arborescente con granos ocluidos de hierro metálico.

Se conocen pocos objetos férreos de estas épocas en la zona geográfica que nos ocupa. El más antiguo es el instrumento indeterminado de Las Terraceras I. Corresponde a uno de los primeros intentos de obtención de instrumentos metálicos en el Bajo Aragón, pero el estudio analítico nos informa del uso de un procedimiento inadecuado.

Un goterón metálico obtenido por fusión, del poblado Monte Catma, corresponde, básicamente, a una fundición gris perlítica con un contenido de $\mathrm{C}_{\text {equiv. }}$ del orden del $4 \%$, según se deduce de su estructura. Dicho goterón es un claro indicio de que en los hornos de la Segunda Edad del Hierro era posible la obtención del metal en fase líquida (con una temperatura de unos $1.550^{\circ} \mathrm{C}$ ). Estas fundiciones serían desechadas en la Protohistoria ya que su elevada dureza y fragilidad las hacían indeseables. En los hornos se buscaba trabajar con una temperatura inferior (aproximadamente $1.100^{\circ} \mathrm{C}$ ) para obtener el metal semifundido con contenidos bajos de carbono.

En el horno, al final del proceso, se obtenía una torta de hierro dulce y escorias; estas últimas se eliminaban mediante martillado en caliente con lo que se conseguía, también, la unión de las masas metálicas. El hierro dulce se conformaba mecánicamente para obtener el objeto deseado que finalmente se carburaba y se sometía a los tratamientos de superficie precisos hasta obtener una estructura superficial que ofreciera las prestaciones requeridas.

\section{CONCLUSIONES}

Se ha estudiado un conjunto minero ibérico que ha permitido una aproximación al conocimiento de estas explotaciones durante la Protohistoria en el levante peninsular.

Los análisis efectuados en los materiales de un horno de reducción inmediato a las minas y en muestras de los escoriales de poblados ibéricos cercanos han posibilitado el conocimiento tipológico de este tipo de instalaciones, de sus características de operación y su comparación con instalaciones similares del contexto celtibérico.

Aunque se presentan los resultados de dos objetos férreos, interesa en la zona la excavación de poblados de esta época y el estudio de sus materiales metálicos.

En conjunto, se verifica la importancia de la siderurgia protohistórica levantina y su correspondencia con las citas de los autores antiguos.

\section{REFERENCIAS}

[1] A. Martín-Costea y G. Ruiz-Zapatero, Rev. Metal. Madrid 16 (1980) 31-40.

[2] C. Cucini-Tizzoni y M. Tizzoni, Le antiche scorie del golfo di Follonica (Toscana): Una proposta di tipología, Ed. Et-Milano, Milán, Italia,1992, pp. 89-210.

[3] G. Ruiz-Zapatero y A. Martín-Costea, Kalathos 2 (1982) 7-32.

[4] E. Sanz-Pérez, I. Ruiz-Bustinza, E. Sanz-SÁnchez, J.L. ENRíQUEZ y J.J. CALONGE, Celtiberia 95 (2001) 33-63.

[5] A. Kolling, Bericht der Staatlichen Denkmalpflege im Saarland 20 (1973) 51-59. 